\title{
Validation of histological diagnostic methods for detecting endothelin $B$ receptor expression
}

\author{
NAOYA FUKUDA, TAKAHIRO TSUCHIKAWA, AKIRA FUKUNAGA, HIROSHI KAWASE, \\ NAOTAKE HOMMA, TORU NAKAMURA, TOSHIAKI SHICHINOHE and SATOSHI HIRANO
}

\author{
Department of Gastroenterological Surgery II, Hokkaido University Graduate School of Medicine, Sapporo, Japan
}

Received November 8, 2013; Accepted December 9, 2013

DOI: 10.3892/or.2014.3031

\begin{abstract}
Pancreatic ductal adenocarcinoma (PDAC) has an extremely poor prognosis. Recently, it was reported that the endothelin $\mathrm{B}$ receptor (ETBR) of tumor endothelial cells prevents antitumor immunity. However, the immunohistochemistry (IHC) conditions required to detect ETBR expression remain unclear. The aim of the present study was to confirm the appropriate conditions for IHC for ETBR using ETBR cDNA and transfectant cells and to assess ETBR expression in PDAC patients. An ETBR-expressing cell was established as an objective positive control and the detectability of ETBR expression was evaluated using several types of anti-ETBR antibodies. ETBR mRNA expression was then studied. Finally, ETBR expression was examined in human PDAC tissue using IHC. As a result, four different anti-ETBR antibodies recognized the cell surface ETBR appropriately. A non-specific reaction was shown in the detection of ETBR in normal human tissues. ETBR mRNA expression was weakly detected only in the adrenal gland. No biologically significant correlation was observed in the ETBR-IHC of human PDAC sections. In conclusion, it is necessary to perform IHC using an appropriate control to assess the tissue expression of ETBR.
\end{abstract}

\section{Introduction}

Pancreatic ductal adenocarcinoma (PDAC) is one of the most difficult-to-treat types of cancer and has an extremely poor prognosis $(1,2)$. The only effective treatment for PDAC is surgical resection. Research and development of chemotherapy and immunotherapy are progressing, but their clinical utility remains insufficient $(1,2)$. To classify the poor prognosis group of PDAC, an immunohistological analysis using paraffin sections from surgical specimens was developed

Correspondence to: Dr Naoya Fukuda, Department of Gastroenterological Surgery II, Hokkaido University Graduate School of Medicine, N-15, W-7, Kita-Ku, Sapporo 060-8638, Japan

E-mail: naoyaf@med.hokudai.ac.jp

Key words: antitumor immunity, endothelin B receptor, immunohistochemistry, pancreatic ductal adenocarcinoma as a new molecular biological method $(3,4)$. Endothelin B receptor (ETBR) is a glycoprotein that consists of 442 amino acid residues and penetrates through the cell membrane seven times (5). It has been reported that ETBR of tumor endothelial cells prevents antitumor immunity. Specifically, ETBR overexpression prevents the endothelial barrier to $\mathrm{T}$ cell homing to tumors in human ovarian cancer (6). It was previously reported in our facilities that the existence of tumor-infiltrating $\mathrm{T}$ lymphocytes in PDAC tissue served as a significant factor related to good prognosis (3). The role of the endothelin axis in a cancer domain other than ETAR of prostate cancer has rarely been studied $(7,8)$. Several studies have examined breast cancer (9), melanoma (10,11), glioblastoma (12), nonsmall cell lung (13), colon (14-16), gastric (17) and squamous cell carcinoma (18). Therefore, we sought to examine ETBR expression in PDAC tissue. However, no studies have reported the conditions needed for immunohistochemistry (IHC) to detect ETBR expression. Therefore, the aim of the present study was to confirm the appropriate conditions required for IHC of ETBR using ETBR cDNA and transfectant cells, as well as to assess ETBR expression in PDAC patients.

\section{Materials and methods}

Human tissue samples, culture cells and xenografts. Frozen normal human tissues were received from the Department of Gastroenterological Surgery II, Hokkaido University Graduate School of Medicine, Sapporo, Japan. PBMCs were obtained from healthy Japanese adult volunteers. HEK293 and 293FT cells were purchased from Invitrogen Corporation (Carlsbad, CA, USA). The human pancreatic cancer cell line PANC-1 was provided by RIKEN (Tsukuba, Japan), PK-9 and PK-45P were from Tohoku University (Sendai, Japan), and SUIT-2 was from Health Science Research Resources Bank (Osaka, Japan). Human pancreatic cancer xenografts were established by subcutaneous injection of these cell lines into female BALB/c-SCID mice.

ETBR subcloning. Total RNA from normal human adrenal gland was extracted by the RNeasy Mini kit (Qiagen, Tokyo, Japan). The cDNA synthesis reaction was performed as previously described (19). ETBR-cDNA was amplified by PCR. Briefly, each $50-\mu 1$ reaction mixture contained $1 \mu \mathrm{l}$ of reverse transcription reaction products, 1 unit of KOD-Plus-DNA 
polymerase, $5 \mu \mathrm{l}$ PCR buffer, $5 \mu \mathrm{l}$ of $2 \mathrm{mM}$ deoxynucleotide triphosphate, $2 \mu \mathrm{l}$ of $25-\mathrm{mM} \mathrm{MgSO}_{4}$ (all from Toyobo, Osaka, Japan), and $1.5 \mu \mathrm{l}$ of each $10-\mu \mathrm{M} 3^{\prime}$ and $5^{\prime}$ primer specific for ETBR (sense, 5'-CGGCTAGCCCTTCTGGAGCAGGTA-3' and antisense, 5'-CGCGGATCCTCAAGATGAGCTGTA-3'). ETBR cDNA was amplified for 30 cycles. Conditions for ETBR PCR were: $94^{\circ} \mathrm{C}$ for $15 \mathrm{sec}, 40^{\circ} \mathrm{C}$ for $30 \mathrm{sec}$ and then $68^{\circ} \mathrm{C}$ for $3 \mathrm{~min}$. All PCR products were electrophoresed in a $2.0 \%$ agarose gel and visualized by ethidium bromide staining. Plasmids expressing ETBR were generated by the PCR amplification of ETBR cDNA and cloning into the NheI and BamHI sites of pcDNA3.1(+) (Invitrogen).

Transfection. Subsequently, pcDNA-3.1(+)-ETBR-IRES-GFP (pETBR) was transfected into 293FT or HEK293 cells using Lipofectamine ${ }^{\circledR} 2000$ (Invitrogen). Transfected cells were incubated at $37^{\circ} \mathrm{C}$ in a $5 \% \mathrm{CO}_{2}$ incubator for $20 \mathrm{~h}$ prior to harvest.

Semi-quantitative reverse transcription-polymerase chain reaction $(R T-P C R)$. Total RNA extraction and cDNA synthesis of normal human tissues were performed as described above. Multiplex PCR was performed using primers specific for ETBR or glyceraldehyde-3-phosphate dehydrogenase (GAPDH). Briefly, each $20-\mu 1$ reaction mixture contained $1 \mu 1$ reverse transcription reaction products, $0.2 \mu 1 \mathrm{Taq}$ DNA polymerase, $4 \mu 1$ reaction buffer (both from Promega, Madison, WI, USA), $0.5 \mu 1$ of 10-mM deoxynucleotide triphosphate, and $0.5 \mu 1$ of each $10-\mu \mathrm{M} 3$ ' and 5' primer specific for ETBR (as described above) and GAPDH (sense, 5'-ACCCCTTCATTGACCTCAACT-3' and antisense, 5'-TGAGTCCTTCCACGATACCAA-3'). ETBR and GAPDH cDNA was amplified for 25, 30, 35 and 40 cycles. Conditions for ETBR and GAPDH PCR were: $95^{\circ} \mathrm{C}$ for $30 \mathrm{sec}$, $50^{\circ} \mathrm{C}$ for $80 \mathrm{sec}$, and then $72^{\circ} \mathrm{C}$ for $5 \mathrm{~min}$. All PCR products were electrophoresed in a $2.0 \%$ agarose gel and visualized by ethidium bromide staining.

Western blot analysis. Western blot analysis was performed to analyze ETBR and GFP protein expression in transfected 293FT and HEK293 cells, normal human tissues, human pancreatic cancer cell lines and xenografts. Briefly, lysates from cells, tissues and xenografts were resolved using $15 \%$ sodium dodecyl sulfate-polyacrylamide gel electrophoresis and then transferred to polyvinylidene fluoride microporous membranes (Millipore, Billerica, MA). Anti-ETBR rabbit polyclonal antibodies (Ab1, 3, 4), mouse monoclonal antibody (Ab2), and anti-GFP and anti- $\beta$-actin mouse monoclonal antibodies were used as the primary antibodies. Peroxidaseconjugated goat anti-rabbit or mouse IgG was used as the secondary antibody (1:10,000 dilution). The detection of bound antibodies was performed using the electrogenerated chemiluminescence (ECL) system (Amersham, Aylesbury, UK).

Flow cytometry. Flow cytometry was performed by FACSCalibur (BD Biosciences, Franklin Lakes, NJ, USA). Briefly, pETBR-transfected 293FT cells [293FT (pETBR)] were harvested by $0.25 \%$ trypsin, and then anti-ETBR antibodies (Ab1-4) were used as the primary antibody. PE-conjugated goat anti-rabbit or mouse IgG (Beckman Coulter, Fullerton, CA, USA) was used as the secondary antibody.
Patients and tissue specimens. Tumor specimens were obtained from the same 80 patients reported previously (3). This examination was carried out with the approval of the Hokkaido University Ethics Committee.

IHC. Immunohistochemical reactions were carried out using the universal immunoenzyme polymer method. Sections were deparaffinized with xylene, rehydrated through a graded series of ethanol/water and treated in a pressure cooker for $20 \mathrm{~min}$. Endogenous peroxidase activity was blocked by 30 -min incubation with $0.3 \%$ hydrogen peroxide in methanol. After washing in TBS-T, specimens were saturated with $10 \%$ normal goat serum [Histofine MAX-PO (Multi) kit; Nichirei Corp., Tokyo, Japan] for $30 \mathrm{~min}$. Sections were then incubated overnight with anti-ETBR antibodies (Ab1-4) at $4^{\circ} \mathrm{C}$. After three additional washes, the sections were incubated with Histofine Simple Stain MAX-PO (Multi) (Nichirei Corp.) for $20 \mathrm{~min}$ at room temperature. Reaction products were observed by incubation for $\sim 5 \mathrm{~min}$ with 3,30-diaminobenzidine tetrahydrochloride (Nichirei Corp.). Sections were counterstained in hematoxylin for $1 \mathrm{~min}$ and then mounted in Marinol (micro slides; Muto-Glass, Tokyo, Japan).

\section{Results}

Establishment of ETBR-expressing cells. Three vectors were constructed as follows (Fig. 1A): pcDNA3.1(+) (pEmpty Vector), pcDNA3.1(+)-IRES-GFP (pGFP), and pcDNA3.1(+) -ETBR-IRES-GFP (pETBR). Four sense and antisense primers were synthesized (Fig. 1B), and the sequence after subcloning was confirmed (data not shown). The amino acid sequence corresponding to the ETBR base sequence was equal to the ETBR sequence described in The National Center for Biotechnology Information (NCBI) (Fig. 1C). Transfection was then performed, and the detectability of ETBR expression by western blot analysis (WB), flow cytometry and IHC was evaluated. On WB, the expression of GFP protein was confirmed in both 293FT (pGFP) and 293FT (pETBR) lysates. For the ETBR protein, bands (100, 37, $25 \mathrm{kDa})$ were shown only in the 293FT (pETBR) lysate by all four ETBR antibodies. The density of the 25-kDa band appeared different with each antibody (Fig. 2). HEK293 cell lysates showed lower density bands of the same size as compared with 293FT. In the normal human tissue sample, specific bands of the same size as the positive control [PC; 293FT (pETBR)] were not observed with all tissue samples. On the other hand, different size bands from PC were found. This tendency was different with each antibody (Fig. 2). On flow cytometry, ETBR expression of 293FT (pETBR) was observed with all antibodies. However, the ratio of ETBR positivity varied by antibody (Fig. 3). On IHC, no immunostaining was observed with the negative control [NC; 293FT (pGFP)] by all antibodies, and immunostaining was found on the membrane surface with the PC by all antibodies. No immunostaining was seen with isotype IgG (Fig. 4). This result remained the same under different $\mathrm{pH}$ conditions (data not shown). In normal human tissues, excluding the adrenal gland, obvious immunostaining such as 293FT (pETBR) was not shown. In the adrenal gland, the staining region (cortex or medulla) and intensity were different with each antibody (Fig. 4). These 
A

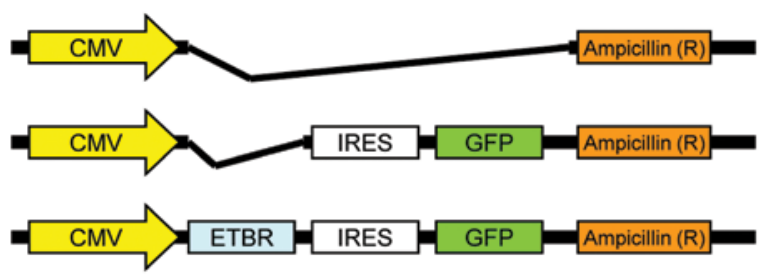

B

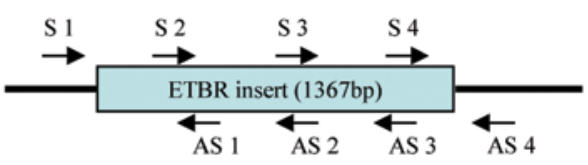

$\mathrm{C}$
1 MQPPPSLCGR ALVALLLACG LLGVWGEERG FPPDKATPLL QTAEIMTPPT KTLWPKGSNA
61 SLARSLAPAE VPKGDRTAGS PPRTISPPPC QGPIEIKETF KYINTVVSCL VFVLGIIGNS
121 TLLRIIYKNK CMRNGPNILV ASLALGDLLH IVIDIPINVY KLLAEDWPFG AEMCKLVPFI
181 QKASVGITVL SLCALSIDRY RAVASWSRIK GIGVPKWTAV EIVLIWVVSV VLAVPEAIGF
241 DITMDYKGS YLRICLLHPV QKTAFMQFYK TAKDWWLFSF YFCLPLAITA FFYTLMTCEM
301 LRKKSGMQIA LNDHLKQRRE VAKTVFCLVL VFALCWLPLH LSRILKLTLY NQNDPNRCEL
361 LSFLLVLDYI GINMASLNSC INPIALYLVS KRFKNCFKSC LCCWCQSFEE KQSLEEKQSC
421 LKFKANDHGY DNFRSSNKYS SS

Figure 1. Subcloning and transfection of ETBR. (A) ETBR-cDNA was synthesized, and pcDNA3.1(+) (pEmpty Vector, upper), pcDNA3.1(+)-IRES-GFP (pGFP, middle) and pcDNA3.1(+)-ETBR-IRES-GFP (pETBR, lower) were constructed. (B) Sequence analyses were performed using 4 sense (S1-4) and 4 antisense (AS1-4) primers. (C) Amino acid sequence determined from the cloning.

staining results changed under different $\mathrm{pH}$ conditions (data not shown).

Analysis of ETBR mRNA expression. The mRNA of normal human tissues was extracted, and semi-quantitative RT-PCR was performed with the ETBR primer. ETBR mRNA expression was weak only in the adrenal gland sample (Fig. 5).

Evaluation of ETBR expression in human PDAC cell lines and tumor specimens. On WB, no specific band of ETBR was shown by two antibodies (Fig. 6A). On IHC, immunostaining was shown only in the adrenal medulla section by Ab1, maintaining consistency between cell cultures and xenografts (Fig. 6B). Thus, it was considered that Ab1 was more suitable for IHC than $\mathrm{Ab} 2$ as almost all immunostaining by Ab2 was shown to be non-specific. Ab1 was then used for IHC of PDAC tumor specimens. No biologically significant staining was shown in the vascular endothelial cells and the pancreatic cancer cells. Some staining was shown in the pancreatic acinar cells and pancreatic islets (Fig. 7).

\section{Discussion}

An appropriate ETBR gene expression system was created, with 293FT (pETBR) as the PC and 293FT (pGFP) as the NC. Although the theoretical molecular weight of ETBR protein is $49.64 \mathrm{kDa}$, the results of $\mathrm{WB}$ revealed several bands of different sizes. Although all the bands (25, 37, 100, $200 \mathrm{kDa})$ of PC differed from the theoretical molecular weight of ETBR, it is appropriate to consider all these bands are ETBR. The PC

\section{HEK293FT HEK 293}

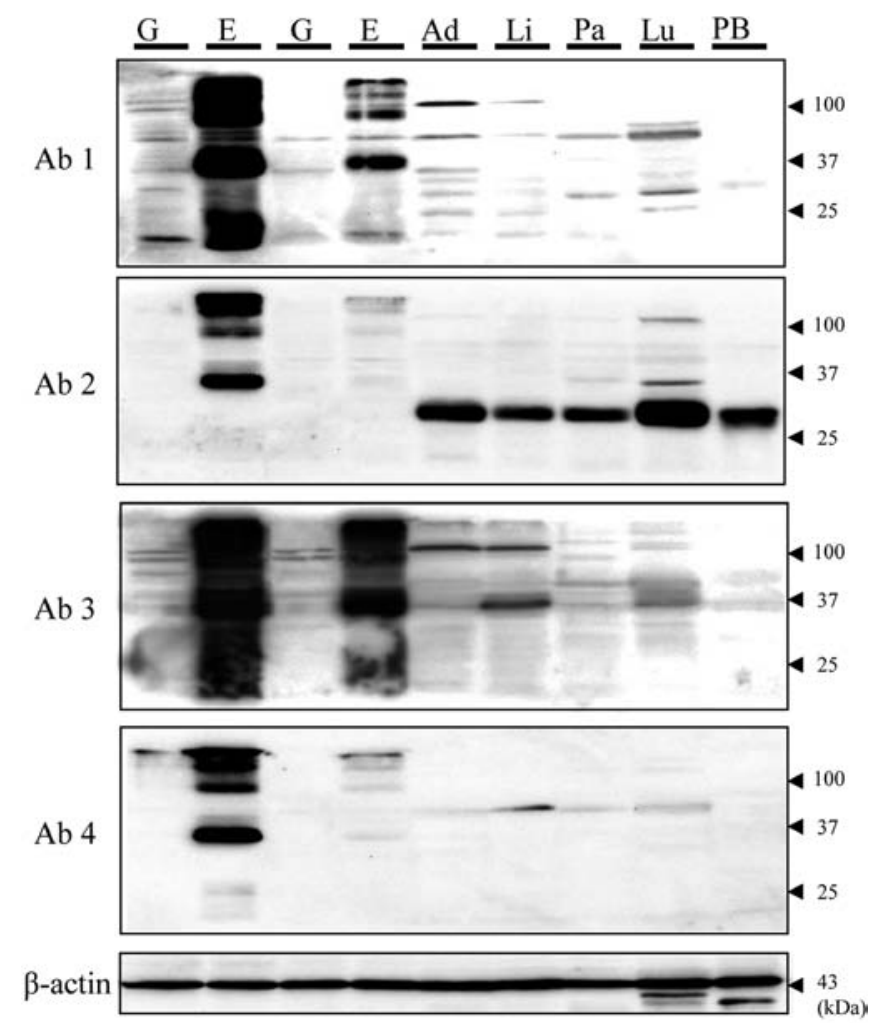

Figure 2. Western blot analysis of HEK293FT, HEK293 transfectants and normal human tissues by 4 ETBR antibodies (Ab1-4). G, 293FT (pGFP); E, 293FT (pETBR); Ad, adrenal gland; Li, liver; Pa, pancreas; Lu, lung; PB, PBMCs. 


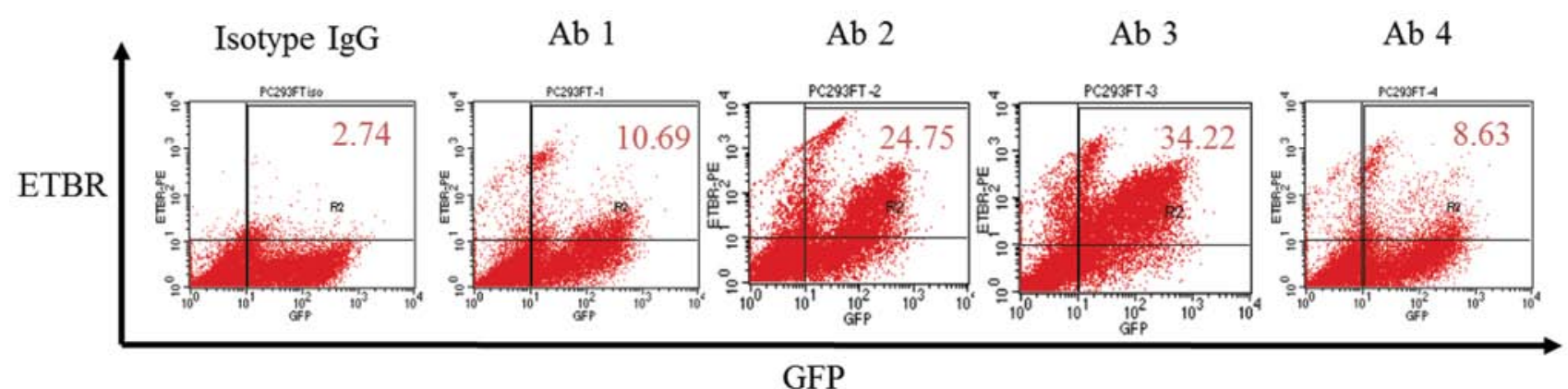

Figure 3. Flow cytometry of HEK293FT transfectants by 4 antibodies. Numbers represent the percentage of GFP and ETBR double-positive cells.

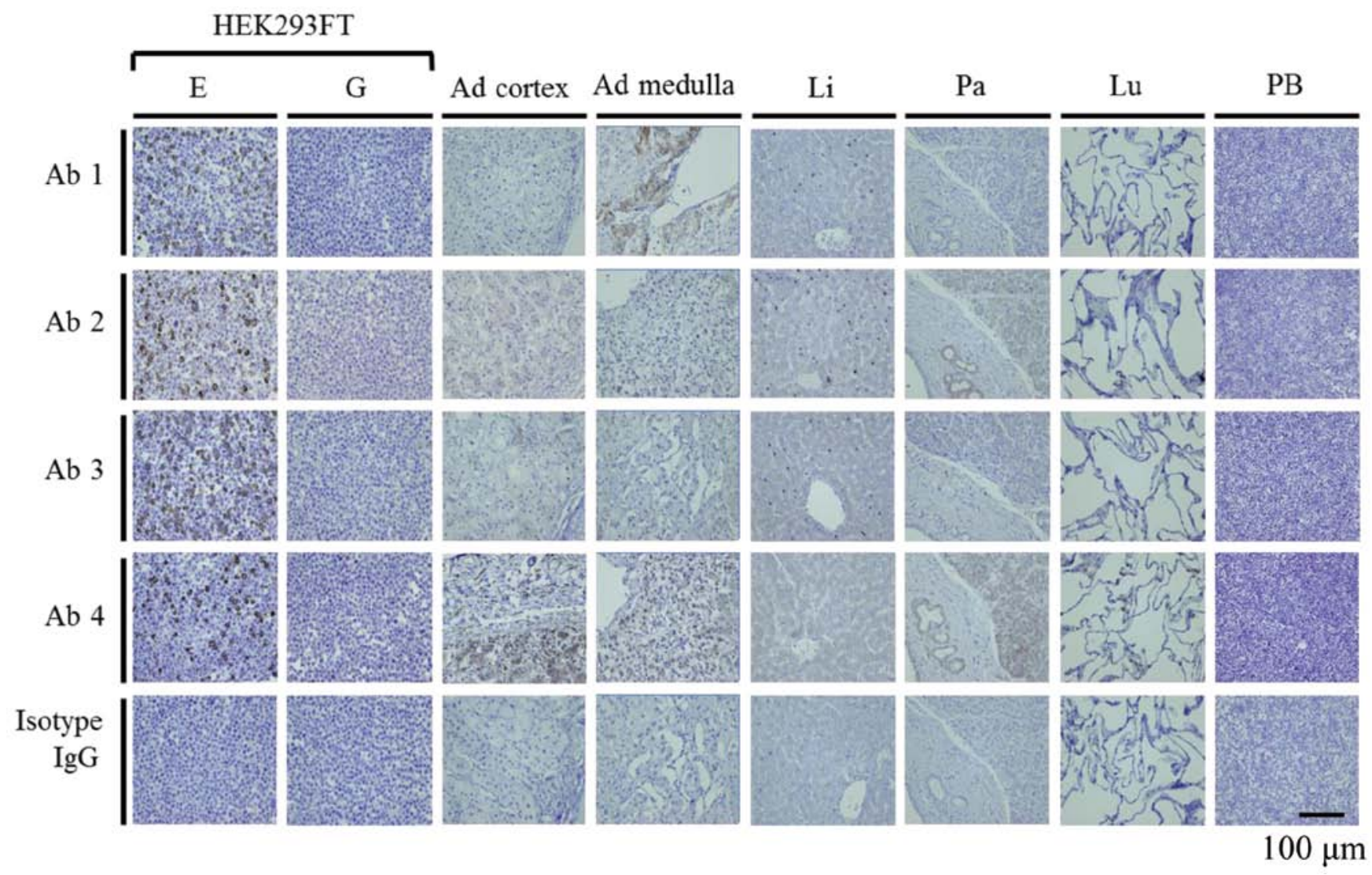

Figure 4. Immunohistochemistry of HEK293FT transfectants or normal human tissues by 4 antibodies. pH 7.0. G, 293FT (pGFP); E, 293FT (pETBR); Ad, adrenal gland; Li, liver; Pa, pancreas; Lu, lung; PB, PBMCs.

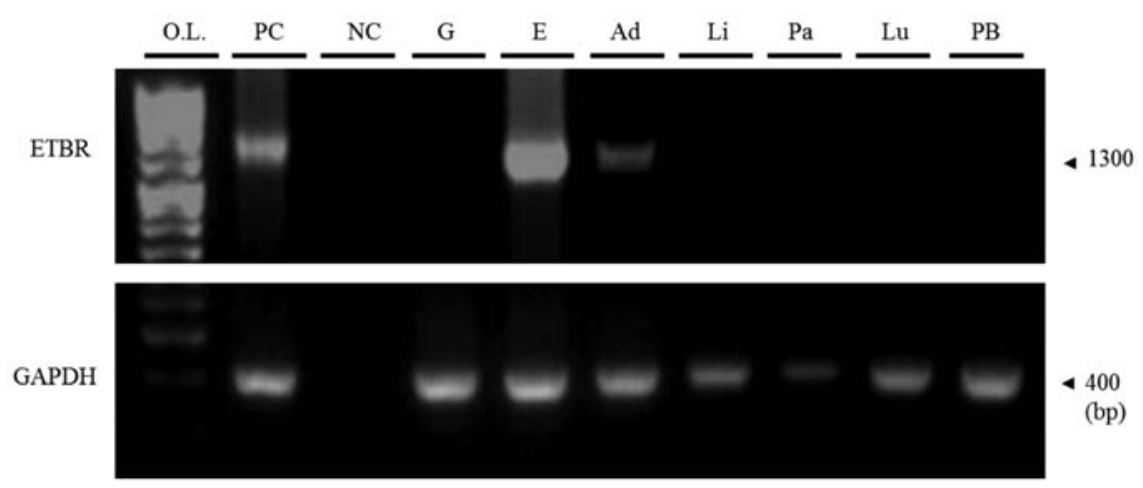

Figure 5. Semi-quantitative reverse transcription-polymerase chain reaction (RT-PCR) of HEK293FT transfectants or normal human tissues by ETBR primers for 35 cycles. Positive control (PC), pcDNA3.1(+)-IRES-GFP-ETBR; negative control (NC), DDW; O.L., one step ladder (marker); G, 293FT (pGFP); E, 293FT (pETBR); Ad, adrenal gland; Li, liver; Pa, pancreas; Lu, lung; PB, PBMCs. 
A
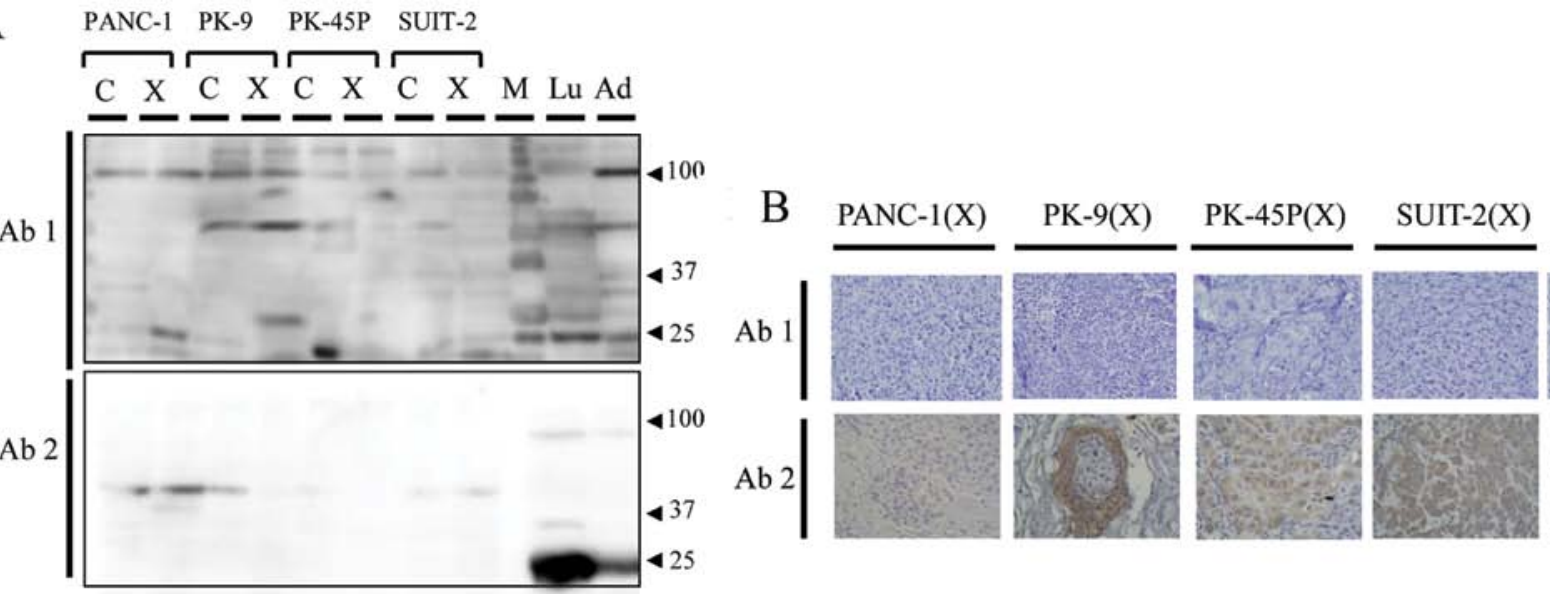

Ad

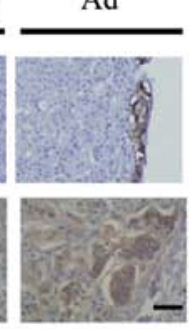

$100 \mu \mathrm{m}$

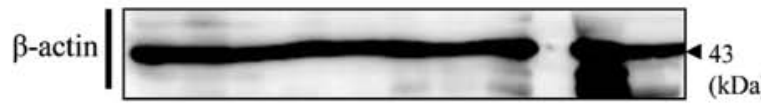

Figure 6. WB and IHC of PDAC cell lines. (A) WB of PDAC cell lines or normal human tissues by two ETBR antibodies. (B) IHC of PDAC cell line xenografts or normal human adrenal gland tissue. C, culture cells; X, xenograft; M, marker; Lu, lung; Ad; adrenal gland; WB, western blot analysis; IHC, immunohistochemistry; PDAC, pancreatic ductal adenocarcinoma; ETBR, endothelin B receptor.

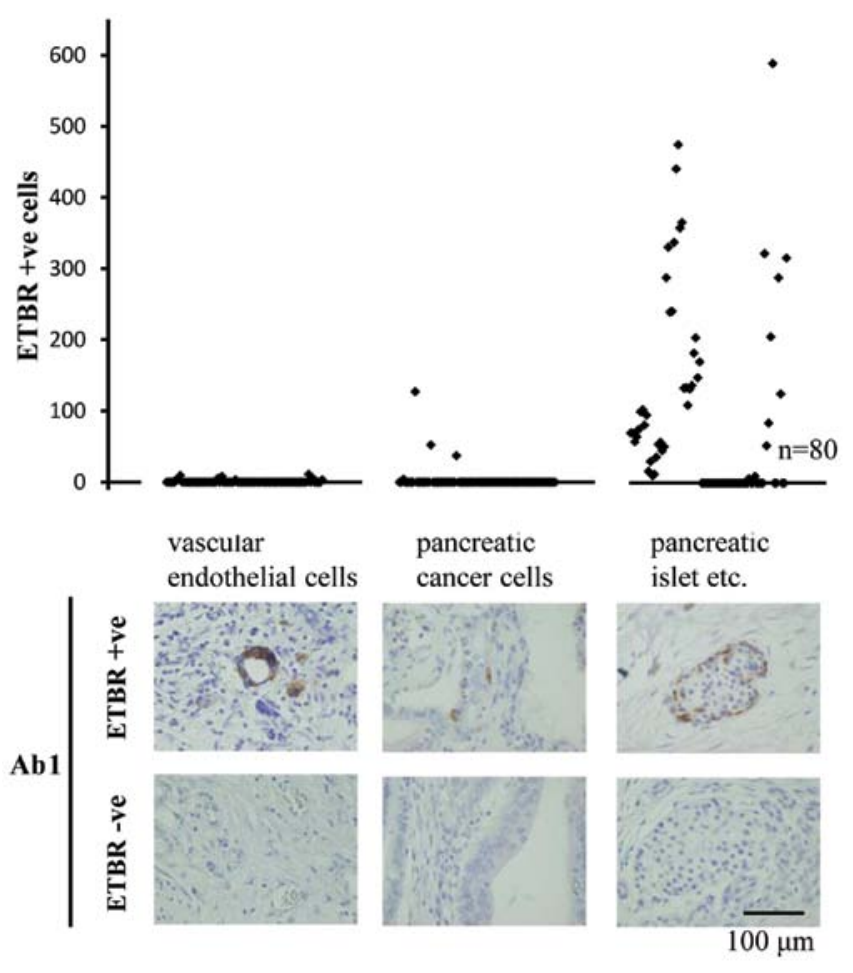

Figure 7. Statistical analysis of IHC of PDAC tumor specimens. Upper panel, positive ETBR expression; lower panel, negative ETBR expression. IHC, immunohistochemistry; PDAC, pancreatic ductal adenocarcinoma; ETBR, endothelin B receptor.

was the NC with the addition of the ETBR gene. Therefore, all bands seen in the PC that were not seen in the NC must be ETBR. Therefore, it is logical that these bands did not vary with the different antibodies.

It has been reported that ETBR can form a homodimer $(20,21)$. In this case, the homodimer shows a size of $100 \mathrm{kDa}$, and polymerization of the homodimer or a combina- tion of the homodimer and other membrane proteins can show a size exceeding $200 \mathrm{kDa}$. If one assumes that although ETBR homodimer is comparatively stable biochemically, monomer is unstable and is cleaved by a specific site during protein extraction, the band of ETBR may also appear with sizes of 25 and $37 \mathrm{kDa}$. Splicing variants $(22,23)$ or glycosylation modification may explain the band size variation from the theoretical molecular weight of ETBR. Since the band of the PC differed from the theoretical molecular weight, examination of the type and concentration of reagent in the case of protein extraction was also performed. It was found that the band of the PC was similarly different from the theoretical figure.

The adrenal gland can serve as a PC of IHC under appropriate conditions due to ETBR-mRNA expression. Of the four ETBR antibodies, Abl was considered to be the most suitable antibody for IHC, as adrenal gland was stained and other tissues were not stained as much (Fig. 4). The examination results for PDAC cell lines suggest that both cultured cells and xenograft tumors may have no biologically significant expression of ETBR. The assessment data on the PDAC tumor specimens demonstrate that both pancreatic cancer cells and vascular endothelial cells have no overexpression of ETBR. Furthermore, it is possible that some intracellular enzymes may contribute to the non-specific staining observed in gland tissue such as pancreatic islets.

Based on the present results, ETBR appears to have no biological significance in PDAC tissue, as non-staining of IHC was regarded as low expression of ETBR.

In IHC, if positive and negative controls with high objectivity are not used, there can be a risk of misleading nonspecific staining for the expression of target molecules.

In conclusion, ETBR expression in human PDAC tissues may not be detected by IHC based on the strict objective controls we have established and, therefore, it was not considered to reflect the grade of malignancy of PDAC. 


\section{Acknowledgements}

The authors thank Dr Satoshi Kondo and Dr Masaki Miyamoto (Department of Gastroenterological Surgery II, Division of Surgery, Hokkaido University Graduate School of Medicine, Sapporo, Japan) for their high level of contribution to this research. The authors would also like to thank Hikaru Shida and Naomi Saito for their technical support in immunohistochemical analyses.

\section{References}

1. Hidalgo M: Pancreatic cancer. N Engl J Med 362: 1605-1617, 2010.

2. Vincent A, Herman J, Schulick R, Hruban RH and Goggins M: Pancreatic cancer. Lancet 378: 607-620, 2011.

3. Fukunaga A, Miyamoto M, Cho Y, et al: $\mathrm{CD}^{+}$tumor-infiltrating lymphocytes together with $\mathrm{CD} 4^{+}$tumor-infiltrating lymphocytes and dendritic cells improve the prognosis of patients with pancreatic adenocarcinoma. Pancreas 28: e26-e31, 2004.

4. Uehara H, Miyamoto M, Kato K, et al: Expression of pigment epithelium-derived factor decreases liver metastasis and correlates with favorable prognosis for patients with ductal pancreatic adenocarcinoma. Cancer Res 64: 3533-3537, 2004.

5. Sakurai T, Yanagisawa M, Takuwa Y, et al: Cloning of a cDNA encoding a non-isopeptide-selective subtype of the endothelin receptor. Nature 348: 732-735, 1990.

6. Buckanovich RJ, Facciabene A, Kim S, et al: Endothelin B receptor mediates the endothelial barrier to $\mathrm{T}$ cell homing to tumors and disables immune therapy. Nat Med 14: 28-36, 2008.

7. Nelson JB, Fizazi K, Miller K, et al: Phase 3, randomized, placebo-controlled study of zibotentan (ZD4054) in patients with castration-resistant prostate cancer metastatic to bone. Cancer 118: 5709-5718, 2012.

8. Miller K, Moul JW, Gleave M, et al: Phase III, randomized, placebo-controlled study of once-daily oral zibotentan (ZD4054) in patients with non-metastatic castration-resistant prostate cancer. Prostate Cancer Prostatic Dis 16: 187-192, 2013.

9. Clézardin P: Therapeutic targets for bone metastases in breast cancer. Breast Cancer Res 13: 207, 2011.
10. Asundi J, Reed C, Arca J, et al: An antibody-drug conjugate targeting the endothelin $\mathrm{B}$ receptor for the treatment of melanoma. Clin Cancer Res 17: 965-975, 2011.

11. Cruz-Muñoz W, Jaramillo ML, Man S, et al: Roles for endothelin receptor B and BCL2A1 in spontaneous CNS metastasis of melanoma. Cancer Res 72: 4909-4919, 2012.

12. Liu Y, Ye F, Yamada K, et al: Autocrine endothelin-3/endothelin receptor B signaling maintains cellular and molecular properties of glioblastoma stem cells. Mol Cancer Res 9: 1668-1685, 2011.

13. Knight LJ, Burrage J, Bujac SR, et al: Epigenetic silencing of the endothelin-B receptor gene in non-small cell lung cancer. Int J Oncol 34: 465-471, 2009.

14. Puglisi MA, Barba M, Corbi M, et al: Identification of Endothelin-1 and NR4A2 as CD133-regulated genes in colon cancer cells. J Pathol 225: 305-314, 2011.

15. Sørby LA, Kleiveland CR, Andersen SN, Bukholm IR and Jacobsen MB: The endothelin axis in the metastatic process of colon carcinoma. Anticancer Res 31: 861-869, 2011.

16. Wang R, Löhr CV, Fischer K, et al: Epigenetic inactivation of endothelin-2 and endothelin-3 in colon cancer. Int J Cancer 132: 1004-1012, 2013.

17. Tao K, Wu C, Wu K, et al: Quantitative analysis of promoter methylation of the EDNRB gene in gastric cancer. Med Oncol 29: 107-112, 2012.

18. Ishimoto $\mathrm{S}$, Wada $\mathrm{K}$, Tanaka $\mathrm{N}$, et al: Role of endothelin receptor signalling in squamous cell carcinoma. Int J Oncol 40: 1011-1019, 2012.

19. Hase R, Miyamoto M, Uehara H, et al: Pigment epitheliumderived factor gene therapy inhibits human pancreatic cancer in mice. Clin Cancer Res 11: 8737-8744, 2005.

20. Evans NJ and Walker JW: Endothelin receptor dimers evaluated by FRET, ligand binding, and calcium mobilization. Biophys J 95: 483-492, 2008

21. Klammt C, Srivastava A, Eifler N, et al: Functional analysis of cell-free-produced human endothelin B receptor reveals transmembrane segment 1 as an essential area for ET-1 binding and homodimer formation. FEBS J 274: 3257-3269, 2007.

22. Elshourbagy NA, Adamou JE, Gagnon AW, Wu HL, Pullen M and Nambi P: Molecular characterization of a novel human endothelin receptor splice variant. J Biol Chem 271: 2530025307, 1996

23. Shyamala V, Moulthrop TH, Stratton-Thomas J and TekampOlson P: Two distinct human endothelin B receptors generated by alternative splicing from a single gene. Cell Mol Biol Res 40: 285-296, 1994 